\title{
Comportamiento de lesiones por accidente de trabajo no mortales y con incapacidad temporal, en personas afiliadas al régimen general de seguridad social en Ecuador. Análisis temporal y territorial en ocupaciones manuales de las industrias manufacturera y de construcción, 2014-20191
}

Behavior of non-fatal occupational injuries with temporary disability among members of Ecuador's general social security system. Temporal and territorial analysis in manual occupations in the manufacturing and construction industry, 2014 - 2019

Comportamento de lesões profissionais não fatais e temporariamente incapacitadas em pessoas filiadas ao sistema geral de segurança social no Equador. Análise temporal e territorial em ocupações manuais das indústrias manufatureiras e de construção, 2014-2019

Antonio Ramón Gómez García

Alywin Ildefonso Hacay Chang León ${ }^{3}$

Sofía Marisol Crespo Vicuña ${ }^{4}$

Karina Estefanía Garzón Quezada

\footnotetext{
${ }^{1}$ Este artículo es resultado parcial del proyecto de investigación Perfil de Siniestralidad Laboral en Ecuador, dirigido por el profesor Antonio Ramón Gómez García y aprobado por el Centro de Investigación de la Universidad Espíritu Santo, Ecuador.

${ }^{2}$ Técnico y magíster en Prevención de Riesgos Laborales. Doctor en Prevención de Riesgos Laborales, Calidad y Medio Ambiente. Observatorio Ecuatoriano de Seguridad y Salud en el Trabajo. Docente - Investigador, Universidad Espíritu Santo. Samborondón, Ecuador. agomezg@uees.edu.ec (iD) https://orcid.org/0000-0003-1015-1753

${ }^{3}$ Ingeniero Industrial. Magíster en Seguridad y Salud Ocupacional. Doctorando en Tecnologías de la Salud y el Bienestar, Universidad Politécnica de Valencia. Docente - Investigador, Universidad Espíritu Santo.Samborondón, Ecuador. ahacay@uees.edu.ec iD https://orcid.org/0000-0002-5383-0951

${ }^{4}$ Médica. Magíster en Seguridad y Salud Ocupacional. Investigadora, Universidad Espíritu Santo. Samborondón, Ecuador. sofiacrespo@uees.edu.ec iD https://orcid.org/0000-0002-2548-6233

${ }^{5}$ Médica. Magíster en Seguridad y Salud Ocupacional. Investigadora, Universidad Espíritu Santo. Samborondón, Ecuador. karinagarzon@uees.edu.ec iD https://orcid.org/0000-0003t-2315-0987
} 


\title{
RESUMEN
}

El análisis de datos cuantitativos de siniestralidad laboral es importante para orientar la gestión preventiva en la República del Ecuador. Se presenta un estudio ecológico exploratorio de la incidencia de lesiones por accidente de trabajo no mortales y con incapacidad temporal, en ocupaciones manuales de las industrias manufacturera y de construcción en 23 provincias del país, para el período comprendido entre 2014 y 2019. Como fuente se utilizaron los registros oficiales de trabajadoras y trabajadores afiliados al régimen general de seguridad social. Se aplicó el programa estadístico SPSS versión 21.0 y Tableau versión 3.0; se agruparon las provincias del Ecuador en cuartiles según el valor de la tasa de incidencia de las lesiones por cada 1.000 personas afiliadas, considerando año y actividad económica. Las tasas de incidencia muestran patrones similares para los dos sectores económicos y un decrecimiento progresivo en el período analizado, con valores semejantes en 2019. Los valores más altos se presentan en las provincias de Azuay, Guayas, Pichincha y Tungurahua. Se concluye que existen importantes diferencias geográficas, lo que demanda estrategias de prevención y disminución de accidentes de trabajo con participación de los equipos interdisciplinarios relacionados, incluyendo terapeutas ocupacionales.

\section{PALABRAS CLAVE}

industria de la construcción, industria manufacturera, accidentes de trabajo, Ecuador

\begin{abstract}
Analyzing quantitative data on occupational accidents is essential to guide preventive management in the Republic of Ecuador. An exploratory ecological study of the incidence of non-fatal and temporary incapacity occupational injuries in manual occupations in the manufacturing and construction industries in 23 provinces is presented for the period 2014-2019. Official registers of workers registered in the general social security system were used as a source. The statistical software SPSS version 21.0 and Tableau version 3.0 were applied; Ecuador provinces were grouped into quartiles according to the value of the incidence rate of injuries per 1,000 registered persons, considering the year and economic activity. Incidence rates show similar patterns for the two economic sectors and a progressive decrease over the period analyzed, with similar values in 2019. The highest values are found in Azuay, Guayas, Pichincha and Tungurahua provinces. It is concluded that there are significant geographical differences, which calls for strategies to prevent and reduce occupational accidents with the participation of related interdisciplinary teams, including occupational therapists.
\end{abstract}

\section{KEY WORDS}

construction industry; manufacturing industry; accidents, occupational; Ecuador 


\section{RESUMO}

A análise de dados quantitativos sobre acidentes de trabalho é importante para orientar a gestão preventiva na República do Equador. Um estudo ecológico exploratório da incidência de acidentes de trabalho não fatais e de invalidez temporária é apresentado em ocupações manuais nas indústrias de manufatureira e de construção em 23 províncias do país, para o período entre 2014 e 2019. Como fonte, foram utilizados os registros oficiais dos trabalhadores filiados ao regime geral de previdência social. Os programas estatísticos SPSS (versão 21.0) e Tableau (versão 3.0) foram aplicados; as províncias do Equador foram agrupadas em quartis, de acordo com o valor da taxa de incidência de lesões por 1.000 afiliados, considerando ano e atividade econômica. As taxas de incidência apresentam padrões semelhantes para os dois setores econômicos e uma diminuição progressiva no período analisado, com valores semelhantes em 2019. Os maiores valores encontram-se nas províncias de Azuay, Guayas, Pichincha e Tungurahua. Conclui-se que existem diferenças geográficas importantes, que demandam estratégias de prevenção e de redução dos acidentes de trabalho com a participação de equipes interdisciplinares relacionadas, incluindo terapeutas ocupacionais.

\section{PALAVRAS-CHAVE}

indústria da construção, indústria manufatureira, acidentes de trabalho, Equador

\section{Introducción}

Los accidentes de trabajo continuan representando un problema de salud pública. Según la Organización Internacional de Trabajo (OIT, 2019), se estima que cada año ocurren más de 374 millones de accidentes de trabajo en el mundo y 7,6 millones en la región de las Américas (Organización Panamericana de la Salud [OPS], 2017). En muchas ocasiones, estos producen incapacidad laboral de carácter temporal o permanente debido a la gravedad de las lesiones que generan, lo que conlleva altos costos sociales, económicos y sanitarios en los países en desarroIlo (OIT, 2012).

Para desarrollar estrategias públicas en seguridad y salud en el trabajo es necesario contar con datos cuantitativos precisos de siniestralidad laboral (Organización Iberoamericana de Seguridad Social
[OISS], 2012; OIT, 2019). Un enfoque de gran utilidad para estudiar los accidentes de trabajo es la distribución espacio-temporal mediante indicadores de morbilidad (Forst et al., 2015; Morassaei et al., 2013). Estos análisis permiten identificar áreas geográficas y grupos de población trabajadora en riesgo para determinadas actividades económicas y ocupaciones laborales similares (Hernández et al., 2016; López et al., 2009); tal información es útil para explorar posibles influencias socioculturales y económicas de cada territorio y controlar sesgos de confusión (Guerra y do Monte, 2016).

En la República del Ecuador, la evolución y tendencia de los accidentes de trabajo ha sido estudiada desde un enfoque geográfico y temporal (Gómez y Suasnavas, 2015; Gómez et al., 2016; 
Gómez et al., 2017). En estas investigaciones se ha observado una tendencia creciente en la incidencia y la concentración en provincias de la región amazónica del país, posiblemente debido al cambio significativo en sus estructuras productivas, asociado al crecimiento económico; al aumento del empleo con cobertura del sistema de seguridad social, y al desarrollo de normativas legales al comienzo y mediados del decenio de 2010 (Gómez et al., 2019).

Sin embargo, como limitaciones de estos estudios se destacan: cortos períodos de análisis, empleo de casos sin notificación oficial de calificación del Instituto Ecuatoriano de Seguridad Social y análisis agrupados en grandes sectores económicos. Una alternativa para contrarrestarlas consistiría en emplear registros oficiales de accidentes de trabajo calificados en períodos más amplios -especialmente de incapacidad temporal, puesto que constituye un indicador importante de los efectos del trabajo en la salud de la población trabajadora afiliada (Benavides et al., 2017)- y restringir los análisis a aquellas actividades más peligrosas, como son la construcción y la industria manufacturera (Takala, 2020).

Los datos administrativos por accidentes de trabajo de la seguridad social ecuatoriana han mejorado a nivel nacional en los últimos años, debido a las regulaciones laborales y los avances en los derechos de protección social (Velásquez, 2016). Esto representa una oportunidad para el análisis y una línea de base para el diseño de programas y políticas en salud laboral. Sin embargo, uno de los aspectos menos conocidos son las incapacidades laborales en ocupaciones de alto riesgo (Varacallo y Knoblauch, 2021). La exposición a una amplia gama de riesgos mecánicos en las ocupaciones manuales muestra mayor probabilidad de ocurrencia de accidentes respecto a otras ocupaciones, por tanto, las lesiones suelen ser más frecuentes y de mayor gravedad, esta última considerada en términos de daños a la salud. Finalmente, un hecho comprobado es la distribución heterogénea de los accidentes de trabajo entre actividades económicas y grupos ocupacionales (López et al., 2009), donde la variabilidad geográfica desempeña una ventaja explicativa de las posibles diferencias, puesto que se encuentra determinada por las condiciones de trabajo y aspectos culturales propios de cada lugar o región (Piha et al., 2013).

Ante las lagunas existentes y el déficit de conocimiento, el objetivo de este estudio fue describir el comportamiento temporal y proporcionar una representación espacial de la incidencia de lesiones por accidente de trabajo no mortales y con incapacidad temporal, en trabajadoras y trabajadores afiliados al régimen general de seguridad social que desempeñan ocupaciones manuales no cualificadas en las industrias de la construcción y la manufactura en la República del Ecuador, para el periodo comprendido entre 2014 y 2019.

\section{Metodología}

Se realizó un estudio ecológico exploratorio y de distribución territorial, considerando como unidades de análisis 23 de las 24 provincias de la República del Ecuador, según la división político-administrativa vigente (Constitución Política del Ecuador/2008); en el análisis fue excluida la provincia de Galápagos por su baja representatividad (4 casos de 
accidentes de trabajo calificados). Los datos de lesiones por accidentes de trabajo consolidados para el periodo de estudio (2014-2019) fueron facilitados por el Seguro General de Riesgos del Trabajo del Instituto Ecuatoriano de Seguridad Social [IESS]; este es el organismo encargado de la calificación individualizada para la prestación económica y de atención médica por daños derivados de accidentes de trabajo en personas afiliadas al sistema de seguridad social del país (IESS, 2016).

Para este estudio se incluyeron aqueIlos casos calificados de lesiones por accidente de trabajo no mortales y que generaron incapacidad temporal en trabajadoras y trabajadores que desempeñan ocupaciones manuales no cualificadas, acaecidos en el lugar habitual de trabajo para las ramas de actividad económica manufacturera y de construcción (15.023 casos). Esta inclusión se justifica por dos motivos: primero, por la importancia del impacto social y económico de días perdidos por incapacidad laboral asociados a la gravedad de la lesión, aunque por limitaciones de la fuente empleada se desconocen la duración y el diagnóstico (Vaquero et al., 2018); segundo, por ser estas las actividades económicas que entrañan condiciones de trabajo más peligrosas para las ocupaciones estudiadas, particularmente, por la exposición a múltiples factores de riesgo de tipo mecánico (Takala et al., 2014). Además, los cuerpos legales en seguridad y salud laboral encargados de prevenir los accidentes de trabajo son comunes para las actividades económicas incluidas en este estudio (Decreto Ejecutivo 2393/1986; IESS, 2016).

Dadas las limitaciones de información de los trabajadores afiliados, para calcular la tasa de incidencia (tasa de incidencia = número de lesiones por accidente de trabajo no mortales con incapacidad temporal / trabajadores afiliados por cada 1.000), se utilizaron las estimaciones de la Encuesta Nacional Empleo, Desempleo y Subempleo de cada año por ocupación laboral (trabajadores no calificados de ocupaciones elementales), actividad económica (construcción - industria manufacturera) y provincia (23 provincias); esta última, empleada la representación territorial. Tal encuesta es aplicada anualmente desde 1990 por el Instituto Nacional de Estadística y Censos del Ecuador [INEC], para estudiar la situación del empleo y caracterizar el mercado de trabajo en personas mayores de 15 años (INEC, 2018).

El comportamiento temporal del período se analizó mediante el cálculo del coeficiente de determinación (R2) y el coeficiente de correlación de Pearson $(r ; p<0.01)$ en los seis años analizados. Finalmente, se calculó el porcentaje de cambio (PC $=[\mathrm{lt} 1-\mathrm{It} 0] / \mathrm{It} 0 \times 100)$ para valorar el aumento o la disminución que experimentaron las tasas de incidencia, dónde It1 fue considerado el último año (2019) y lt0 el primer año (2014). Se tomó el 2014 como punto de referencia por ser el primer año con datos disponibles en el Seguro General de Riesgos del Trabajo.

El programa estadístico utilizado fue SPSS (Statistical Package for Social Science) versión 21.0 y Tableau versión 3.0 para la representación en mapas temáticos de la República del Ecuador, agrupando las provincias en cuartiles según el valor de la tasa de incidencia por año y actividad económica.

Aspectos éticos. Este estudio utilizó datos secundarios anónimos de los trabajadores y fueron facilitados con fines 
investigativos por la Dirección del Seguro General de Riesgos del Trabajo al Observatorio Ecuatoriano de Seguridad y Salud en el Trabajo, por lo que no requirió de la aprobación por parte de un Comité de Ética.

\section{Resultados}

La figura 1 muestra la evolución del número de trabajadores afiliados, casos de lesiones por accidente de trabajo no mortales con incapacidad temporal y tasas de incidencia, por actividad económica. La construcción muestra descensos pronunciados de afiliados (17.873 en 2014 y 7.351 en 2019) y casos de lesiones (1.249 en 2014 y 219 en 2019) en los seis años de estudio. En cuanto a los afiliados de la actividad económica de manufactura, presenta oscilaciones con incrementos progresivos entre 2014 (36.097), 2016 (47.749) y hasta 2017 (34.917), con una caída considerable y

Figura 1. Evolución de afiliados, casos de lesiones por accidente de trabajo no mortales con incapacidad temporal y tasas de incidencia, según actividad económica. República del Ecuador, 2014-2019.

\section{Construcción}
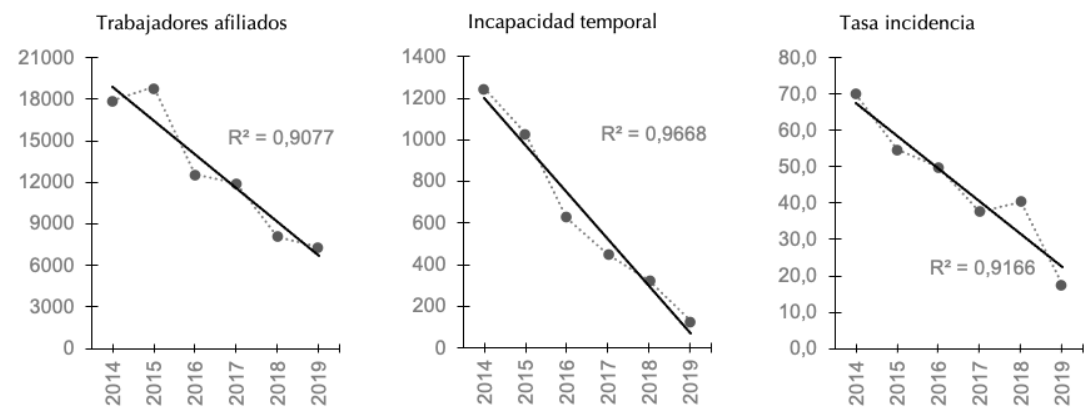

\section{Manufactura}
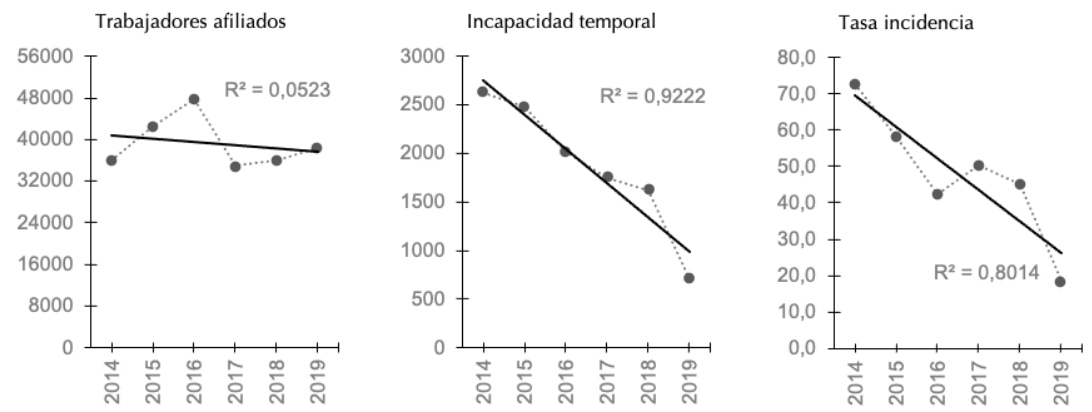

Fuente: elaboración propia a partir de datos del Seguro General de Riesgos del Trabajo y la Encuesta Nacional Empleo, Desempleo y Subempleo. 
aumentos leves en los dos años posteriores, así como tendencias paulatinas a la disminución en los casos de lesiones con mayor decrecimiento entre 2018 (1.625) y 2019 (710).

En general, las tasas de incidencia para ambas actividades económicas muestran patrones similares y se observa un decrecimiento progresivo en el período analizado, con valores semejantes en 2019 por cada 1.000 trabajadores afiliados en la construcción $(17,5)$ y manufactura $(18,5)$. Sin embargo, la tendencia en las tasas difiere, en construcción fue negativa y fuerte $\left(R^{2}=0,924\right)$ y estadísticamente significativa a los trabajadores afiliados y casos de accidentes de trabajo no mortales con incapacidad temporal $(r=0,968 ; p<0,01)$. Por el contrario, en la manufactura fue negativa y débil $\left(R^{2}=0,036\right)$, sin encontrarse asociaciones estadísticamente significativas ( $r=0,190 ; p>0,01)$.

En la figura 2 se puede observar la tasa de incidencia en cuartiles (escala de grises) por año y actividad económica según provincia de la República del Ecuador entre 2014 a 2019. Los valores más altos en las tasas de incidencia para la actividad económica de construcción corresponden a la provincia de Pichincha, que se encuentra siempre de forma consistente en el tercer $(30,6-73,5)$ y cuarto cuartil $(\geq 73,5)$ hasta 2018 . Las provincias de Napo y Zamora Chinchipe también se ubican en los mismos cuartiles, aunque hasta 2017. Por otro lado, Azuay, Bolívar y Tunguragua destacan igualmente por encontrarse entre las provincias con las más altas tasas entre los cuartiles segundo al cuarto.

En cuanto a la actividad económica de manufactura, destacan especialmente las provincias de Azuay, Guayas, Pichincha y Tunguragua con las más altas tasas de incidencia y de forma permanente en el tercer $(31,4-61,7)$ y cuarto cuartil $(\geq 61,8)$ hasta 2018 . Las menores tasas para ambas actividades económicas en el período de estudio corresponden a las provincias de la región amazónica.

Finalmente, solo en algunas provincias del país se observa un decremento significativo en el porcentaje de cambio de las tasas de incidencia desde 2014 a 2019. En la figura 3, en color gris claro se representan aquellas provincias sin cambios porcentuales. Sin embargo, Manabí (+447), Tunguragua $(68,4 \%)$ y Esmeraldas (65,1\%) muestran incrementos en la construcción $y$, aunque con menor incremento, la provincia de Los Ríos $(+3,8 \%)$ en manufactura. 
Figura 2. Distribución de las tasas de incidencia de lesiones con incapacidad temporal, según actividad económica y provincia. República del Ecuador, 2014-2019.

\section{Construcción}
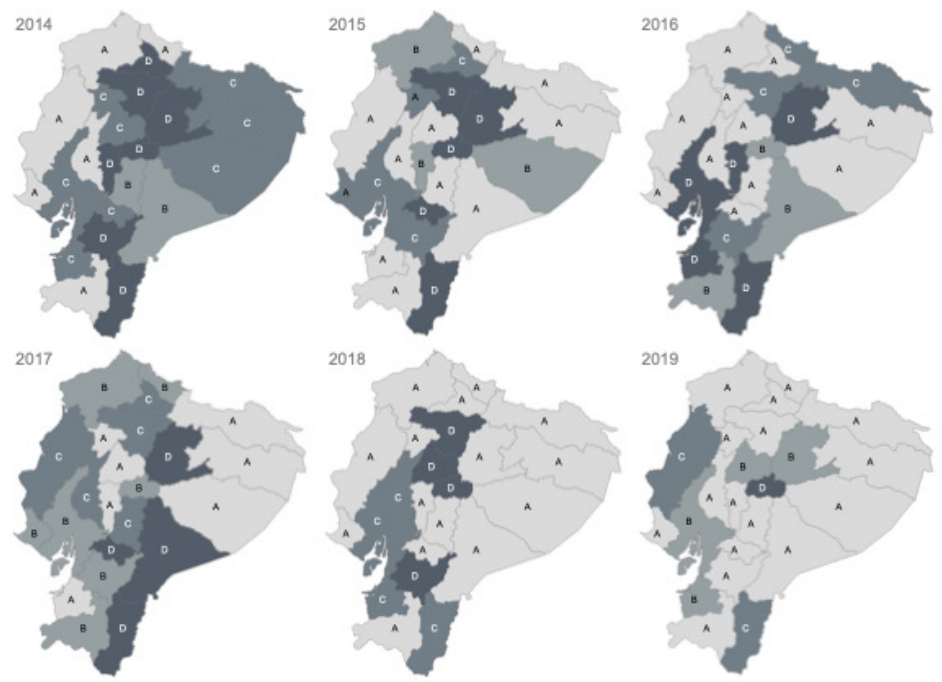

Tasa de incidencia ( $x 1000)$

$\begin{array}{lllll}\text { A } & \leq 11,8 & \text { B } 12,9-30,5 & \text { C } 30,6-73,5 & \text { D } \geq 73,5\end{array}$

\section{Manufactura}
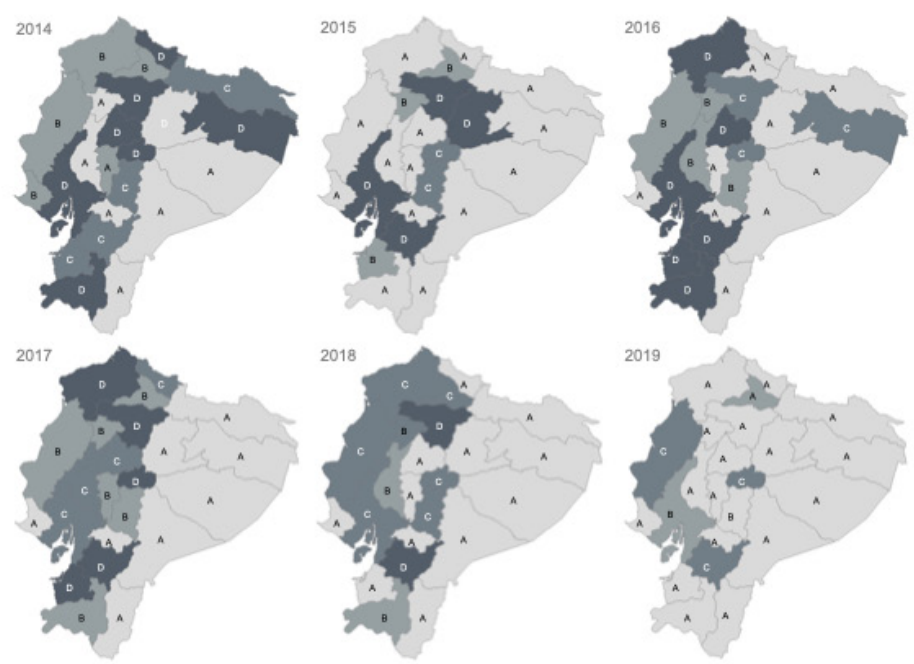

$\begin{array}{lllll}\text { A } \leq 12,9 & \text { B } 13,0-31,3 & \text { C } 31,4-61,7 & \text { D } 261,8\end{array}$

Fuente: elaboración propia a partir de datos del Seguro General de Riesgos del Trabajo y la Encuesta Nacional Empleo, Desempleo y Subempleo. 
Figura 3. Porcentaje de variación de las tasas de incidencia de lesiones con incapacidad temporal, según actividad económica y provincia. República del Ecuador, 2019 vs. 2014.

\section{Construcción}

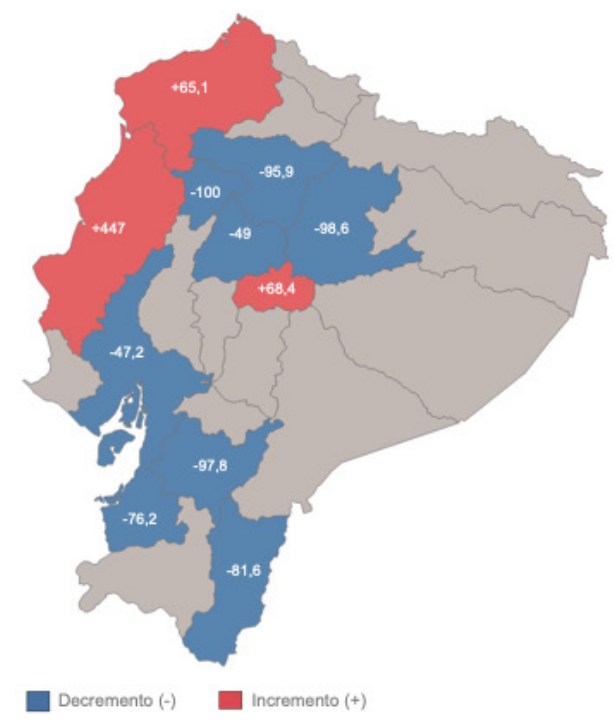

\section{Manufactura}

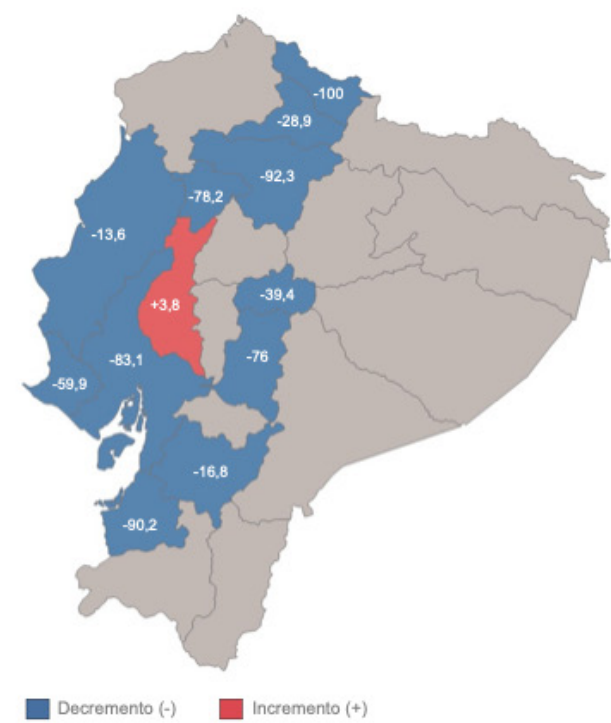

Fuente: elaboración propia a partir de datos del Seguro General de Riesgos del Trabajo y la Encuesta Nacional Empleo, Desempleo y Subempleo. 


\section{Discusión}

En general, los resultados muestran tendencias importantes a la disminución en las tasas de incidencia para las actividades económicas en la mayoría de las regiones del país. Este es el primer estudio que analiza datos nacionales de lesiones por accidente de trabajo no mortales con incapacidad temporal de ocupaciones manuales no cualificadas en actividades económicas peligrosas, para identificar en mapas aquellas provincias con mayor tasa de incidencia en un período amplio (2014 a 2019) en la República del Ecuador.

Nuestros resultados son similares a otros estudios que analizan esta problemática en períodos amplios de tiempo (Benavides et al., 2017; Izadi et al., 2019). No obstante, aunque los descensos observados revelan, a priori, un panorama optimista, podrían estar influenciados por el desempleo. No podemos obviar el posible impacto de la crisis económica en el país. La tasa de desempleo aumentó de $3,4 \%$ a $3,8 \%$ entre 2014 y 2019 (Comisión Económica para América Latina y el Caribe [CEPAL], 2021), lo que podría explicar el descenso progresivo de las tasas de incidencia. Desde un punto de vista epidemiológico, el denominador de la tasa de incidencia influye directamente en el resultado.

Por otra parte, la subnotificación de los accidentes de trabajo podría contribuir a sesgos en las estadísticas oficiales (subregistro) y a la aparente disminución en las tasas de incidencia observadas (Orellana et al., 2021). En la República del Ecuador, un reciente estudio estima la subnotificación potencial de accidentes de trabajo no mortales entre el $81 \%$ y el $96 \%$ (Valenzuela et al., 2020). Este problema es similar en la mayoría de los países de Lationamérica y es particularmente más notorio en pequeñas empresas de las actividades económicas industriales y de construcción, debido a la ausencia de cultura de seguridad o al desconocimiento de las prestaciones por contingencias profesionales (Dong et al., 2011; Moore et al., 2013; Wuellner et al., 2016). En este sentido, sería fundamental la realización de campañas para promover la prevención y el reporte de accidentes de trabajo en las empresas de todas las provincias del país, lo que a su vez supondría una mejora en las condiciones de trabajo.

En cualquier caso, en los últimos años se puede constatar que el riesgo de lesión por accidentes de trabajo ha disminuido en la mayoría de las provincias. Aunque los resultados no sean conclusivos, sería recomendable apuntar a estudios más específicos para conocer el estado de la incapacidad temporal en Ecuador desde una perspectiva interdisciplinar (Beltrán et al., 2021). Así mismo, este y otros estudios sugieren la necesidad de mejorar los sistemas de información estadística y vigilancia contínua de los daños a la salud relacionados con el trabajo (Guzmán et al., 2017; OISS, 2014). Como es probable que el año 2020 marque un hito histórico en la evolución de la siniestralidad laboral, debería analizarse por separado en futuras investigaciones (Hacay y Gómez, 2021).

En cuanto a las fortalezas del estudio, los cálculos de las tasas de incidencia fueron ajustados considerando como denominador la población trabajadora expuesta, precisando las estimaciones presentadas para cada una de las actividades económicas según provincias. Sin embargo, además de las limitaciones 
propias en el empleo de datos secundarios (Benavides y Serra, 2003), existen otros factores determinantes en la ocurrencia de los accidentes de trabajo que deberían estudiarse más a fondo en posteriores investigaciones para conocer con mayor especificidad la población en riesgo y la situación de vulnerabilidad en las regiones del país. Por ejemplo, sería de especial interés analizar la siniestralidad laboral estratificando por sexo, grupos de edad y otros determinantes sociales y laborales para la comparabilidad entre provincias. Por otro lado, considerar por separado las lesiones con incapacidad temporal y permanente (parcial o total) -y con ello la gravedad de las mismas-, es una buena estrategia para medir la siniestralidad laboral (López et al., 2009).

\section{Conclusiones}

Para la mayoría de las provincias de la República del Ecuador, en los últimos años es notable la disminución de las tasas de incidencia de lesiones por accidentes de trabajo con incapacidad temporal en ocupaciones manuales no cualificadas de las industrias manufacturera y de construcción. Sin embargo, se han podido observar importantes diferencias geográficas, donde la magnitud del problema es más evidente en ciertas provincias del país. Es posible que la crisis económica haya incidido de forma diferente por regiones; esta y otras características propias de cada provincia tendrán que ponerse a prueba en futuros estudios.

Los resultados de esta investigación podrían ser considerados para la formulación de programas y estrategias específicas de seguridad y salud en el trabajo, orientados a la prevención y la disminución de accidentes de trabajo en las actividades económicas manufacturera y de construcción. Asimismo, podrían ser utilizados por profesionales que trabajan en este campo, incluyendo terapeutas ocupacionales, para promover la prevención de lesiones y la promoción de la salud en los lugares de trabajo.

\section{Financiación}

Este artículo es resultado de un proyecto realizado con fondos de la Universidad Espíritu Santo y la colaboración de la Dirección General de Riesgos del Trabajo del Instituto Ecuatoriano de Seguridad Social. 


\section{Referencias}

Beltrán, J. A., Valero-Pacheco, I. C., Hernández, M. A., Penagos, L. F. y Perdomo, A. F. (2021) La incapacidad temporal como beneficio de la seguridad social: una revisión de literatura. Revista Ocupación Humana, 21 (1), 42-57. https://doi.org/10.25214/25907816.1032

Benavides, F. G. y Serra, C. (2003). Evaluación de la calidad del sistema de información sobre lesiones por accidentes de trabajo en España. Archivos de Prevención de Riesgos Laborales, 6(1), 26-30.

Benavides, F., Zaballa, E., Sánchez, A. y Porras, D. (2017). Incidencia de la incapacidad temporal por contingencia común en España según la actividad económica de la empresa. Archivos de Prevención de Riesgos Laborales, 20(1), 14-25. https://dx.doi. org/10.12961/aprl.2017.20.01.3

Comisión Económica para América Latina y el Caribe [CEPAL] (2021). CEPALSTAT. Base de datos y publicaciones científicas. CEPAL. https://estadisticas.cepal.org/cepalstat/ portada.html

Dong, X.S., Fujimoto, A., Ringen, K., Stafford, E., Platner, J. W., Gittleman, J. L. y Wang, X. (2011). Injury underreporting among small establishments in the construction industry. American Journal of Industrial Medicine, 54(5), 339-349. https://doi.org/10.1002/ ajim.20928

Forst, L., Friedman, L., Chin, B. y Madigan, D. (2015). Spatial clustering of occupational injuries in communities. American Public Health Association, Suppl 3, S526-S533. https://doi.org/10.2105/AJPH.2015.302595
Guerra, A. y do Monte, A. (2016). Severe occupational accidents and productive activities in health administrative regions in Pernambuco: an analysis based on the identification of local productive cluster. Revista Brasileira de Saúde Ocupacional, 41, e15. https://doi.org/10.1590/2317-6369000115215

Gómez, A. R. y Suasnavas, P .R. (2015). Incidencia de accidentes de trabajo declarados en Ecuador en el período 2011-2012. Ciencia \& trabajo, 17 (52), 49-53. https://dx.doi. org/10.4067/S0718-24492015000100010

Gómez, A. R., Algora, A. F., Suasnavas, P. R., Silva, M. G. y Vilaret, A. (2016). Notificación de accidentes de trabajo y posibles enfermedades profesionales en Ecuador, 2010-2015. Ciencia \& trabajo, 18 (57), 166-172. http://dx.doi.org/10.4067/S0718$\underline{24492016000300166}$

Gómez, A. R., Merino, P., Tapia, O., Espinoza, C. y Echeverría, M. A. (2017). Epidemiología de accidentes de trabajo en Ecuador basado en la base de datos de la Seguridad Social en los años 2014-2016. Scientifica, 15 (2), 14-18.

Gómez, A. R., Suasnavas, P. R. y Argüello, I. F. (2019) Siniestralidad laboral, crecimiento económico y políticas públicas en Ecuador: 2006-2016. Universidad, Ciencia y Tecnología, 23, 93. https://uctunexpo.autanabooks. com/index.php/uct/article/view/144/141

Guzmán, O. B., Oceguera, A. y Contreras, M. I. (2017) Estrategia Iberoamericana de Seguridad y Salud en el Trabajo: políticas públicas para un trabajo decente. Medicina y Seguridad en el Trabajo, 63(246), 4-17. 
Hacay, A.I. y Gómez, A. R. (2021). Efecto 2020 en la siniestralidad laboral de la República del Ecuador: un año atípico por COVID-19. Revista Colombiana de Salud Ocupacional, 11 (1), e-7831. https://doi.org/10.18041/2322-634X/rcso.1.2021.7381

Hernández, A., Díaz, D., Vilcarromero, S. y Santero, M. (2016). Distribución espacial de los accidentes y enfermedades relacionados con el trabajo en el Perú, 2012-2014. Revista Peruana de Medicina Experimental y Salud Pública, 33 (1), 106-112. https://dx. doi.org/10.17843/rpmesp.2016.331.2013

Instituto Ecuatoriano de Seguridad Social [IESS] (2016). Resolución C.D. n. ${ }^{\circ}$ 513. Reglamento del Seguro General de Riesgos del Trabajo. Ecuador.

Instituto Nacional de Estadísticas y Censos [INEC] (2018). Encuesta Nacional Empleo, Desempleo y Subempleo (ENEMDU). INEC. https://www.ecuadorencifras.gob.ec/enemdu-2021/

Izadi, N., Aminian, O. y Esmaeili, B. (2019). Occupational accidents in Iran: Risk factors and long term trend (2007-2016). Journal of research in health sciences, 19 (2), e00448. https://www.ncbi.nlm.nih.gov/pmc/articles/PMC7183544/pdf/jrhs-19-e00448.pdf

López, M., Martínez, J. M., Castejón, E. y Benavides, F. G. (2009). Comparación de las lesiones no mortales por accidente de trabajo por Comunidades Autónomas en España (1994-2004). Gaceta Sanitaria, 23 (6), 489-495.

Morassaei, S., Breslin, F. C., Ibrahim, S. A., Smith, P. M., Mustard, C. A., Amick, B. C., Shankardass, K. y Petch, J. (2013). Geographic variation in work injuries: A multilevel analysis of individual-level data and area-level factors within Canada. Annals of
Epidemiology, 23 (5), 260-266. https://doi. org/10.1016/j.annepidem.2013.03.008

Moore, J. T., Cigularov, K. P., Sampson, J. M., Rosecrance, J. C. y Chen, P.Y. (2013). Construction workers' reasons for not reporting work-related injuries: An exploratory study. International journal of occupational safety and ergonomics, 19 (1), 97-105. https://doi. org/10.1080/10803548.2013.11076969

Organización Iberoamericana de Seguridad Social [OISS] (2012). Recopilación de los principales indicadores de siniestralidad laboral y enfermedad ocupacional utilizados en Iberoamérica. http://www.oiss.org/ estrategia/IMG/pdf/Informe sobre siniestralidad.pdf

Organización Iberoamericana de Seguridad Social [OISS] (2014). Estrategia Iberoamericana de Seguridad y Salud en el Trabajo 2015-2020. OISS.

Organización Internacional del Trabajo [OIT] (2012). Estimating the economic costs of occupational injuries and illnesses in developing countries: Essential information for decision-makers. OIT.

Organización Internacional del Trabajo [OIT] (2019). Safety and health at the heart of the future of work: Building on 100 years of experience. OIT.

Organización Panamericana de la Salud [OPS] (2017). Healt in the Americas 2017. Regional outlook and country. OPS-OMS.

Orellana, C., Kreshpaj, B., Burstrom, B., Davis, L., Frumento, P., Hemmingsson, T., Johansson, G., Kjellberg, K., Wegman, D. H. y Bodin, T. (2021). Organisational factors and under-reporting of occupational injuries in Sweden: A population-based study using capture-recapture methodology. Occupa- 
tional and Environmental Medicine, 78 (10), 745-752. https://doi.org/10.1136/oemed-2020-107257

Piha, K., Laaksonen, M., Martikainen, P., Rahkonen, O. y Lahelma, E. (2013). Socio-economic and occupational determinants of work injury absence. European journal of public health, 23 (4), 693-698. https://doi. org/10.1093/eurpub/cks162

Presidencia de la República (1986). Decreto Ejecutivo 2393. Reglamento de Seguridad y Salud de los Trabajadores y Mejoramiento del Medio Ambiente de Trabajo. Ecuador.

Pueblo Ecuatoriano (2008).Constitución Política del Ecuador. Registro Oficial n. ${ }^{\circ} 1$ del 20 de octubre de 2008.

Takala, J., Hämäläinen, P., Saarela, K. L., Yun, L. Y., Manickam, K., Jin, T. W., Heng, P., Tjong, C., Kheng, L. G., Lim, S. y Lin, G. S. (2014). Global estimates of the burden of injury and illness at work in 2012. Journal of occupational and environmental hygiene, 11 (5), 326-337. https://doi.org/10.1080 /15459624.2013.863131

Takala, J. (2020). Burden of injury due to occupational exposures. In U. Bültmann, J. Siegrist (eds) Handbook of disability, work and health. Handbook Series in Occupational Health Sciences (pp.105-126). Springer. https://doi. org/10.1007/978-3-030-24334-0_5

Vaquero, M., Álvarez, E. y Romero, M. (2018). Influencia de las condiciones de trabajo sobre la incapacidad temporal por contingencias comunes. Atención Primaria, 50 (4), 238-246.
Varacallo, M. y Knoblauch, D. K. (2021). Occupational injuries and workers' compensation management strategies. In StatPearls. StatPearls Publishing. https://www.ncbi.nlm. nih.gov/books/NBK470372/

Velásquez, M. D. (2016). Regulación del mercado de trabajo y protección social en países de América Latina. Serie Políticas Sociales Number 218. CEPAL y Coorperación Alemana. https://repositorio.cepal.org/bitstream/handle/11362/40770/1/S1600024 es.pdf

Valenzuela, R., Bravo, M. y Gómez, A.R. (2020). Subregistro de accidentes de trabajo en Ecuador: nuevas evidencias, limitaciones y prioridades. Universidad, Ciencia y Tecnología, 24 (101), 33-40.

Wuellner, S. E, Adams, D. A. y Bonauto, D.K. (2016). Unreported workers' compensation claims to the BLS survey of occupational injuries and illnesses: Establishment factors. American journal of industrial medicine, 59 (4), 274-289. https://doi.org/10.1002/ ajim. 22563 